\title{
Dogs: Active Role Model for Cancer Studies-A Review
}

\author{
Sana Munaf Hawai ${ }^{1,2}$, M. Al-Zayer ${ }^{1,2}$, Mohammed M. Ali ${ }^{1,2}$, Yuanjie Niu, ${ }^{3,4,5}$, A. Alawad ${ }^{6}$, M. Aljofan ${ }^{2}$, \\ A. Aljarbou ${ }^{7,8}$, S. Altuwaijri ${ }^{1,2,3,4,5,6,7}$ \\ ${ }^{1}$ Saad Research and Development Center, Clinical Research Laboratory, SAAD Specialist Hospital, Al Khobar, Saudi Arabia; \\ ${ }^{2}$ College of Pharmacy, Qassim University, Qassim, Saudi Arabia; ${ }^{3}$ Tianjin Institute of Urological Surgery, Tianjin Medical Univer- \\ sity, Tianjin, China; ${ }^{4}$ Department of Surgery, University of Michigan, Medical Center, Ann Arbor, USA; ${ }^{5}$ George Whipple Lab for \\ Cancer Research, Departments of Pathology, Urology and Oncology, University of Rochester Medical Center, Rochester, USA; \\ ${ }^{6}$ King Abdulaziz City of Science and Technology, Riyadh, Saudi Arabia; ${ }^{7}$ College of Applied Medical Science, Qassim University, \\ Qassim, Saudi Arabia; ${ }^{8} \mathrm{Al}$ Ghad International Medical Science Colleges, Riyadh, Saudi Arabia. \\ Email: sanahawai@gmail.com
}

Received April 20 ${ }^{\text {th }}, 2013$; revised May 22 ${ }^{\text {nd }}, 2013$; accepted May 30 ${ }^{\text {th }}, 2013$

Copyright (C) 2013 Sana Munaf Hawai et al. This is an open access article distributed under the Creative Commons Attribution License, which permits unrestricted use, distribution, and reproduction in any medium, provided the original work is properly cited.

\begin{abstract}
Many studies have been done and many results have been established for studying cancers in human and the ways of treating it. However, one thing that remains relevant is the study model that is used to diagnose, cure or conclude treatment methods for human cancers. The scientists have tried some ways to link the data and tried to analyze the malicious disease in various animal models in order to solve the problem for humans. Out of all the models, scientists have preferred dogs as the most suitable model and conducted studies on them. Our article will review the reason for preferences given to dog as a study model and what the previous studies have tried to conclude by considering the dreaded disease in dogs. Our article has focused on most of the recent observations and tried to elucidate the reasons/preferences for studying cancer disease in dogs (scientific name; Canis Lupus familiaris). We will also talk about the idea of comparative oncology programs that many centers adapt in order to study the disease called cancer.
\end{abstract}

Keywords: Cancer; Models; Dogs; Comparative; Oncology

\section{Introduction}

The most common type of tumor in unspayed female dogs is the mammary tumor especially between the ages of five to 10 years. Some male dogs do develop this type of breast cancer, but due to the hostility of this type of breast cancer, the prognosis is not good. Mammary tumors in dogs range in size and often grow quickly with an irregular shape. These malignant tumors can also root bleeding and ulceration [1]. There were an estimated 12.7 million cancer cases around the world reported in 2008, out of which 6.6 million cases were in men and 6.0 million in women. This number is expected to increase to 21 million by 2030 [2]. According to the statistics in 2011, 230,480 new cases of invasive breast cancer are estimated to be diagnosed in women in the US, along with 57,650 new cases of non-invasive (in situ) breast cancer [3].

Breast cancer being the most common cancer in women, is responsible for almost $20 \%$ of all cancer deaths in women. Increase in awareness and routine mammograms has helped women to get diagnosed in earlier stages of breast cancer thereby increasing the chances of curing it. The statistics state that for every 100 women, one man is also diagnosed with this disease. The disease is more common in women above 40 . It is also more frequent in women of a higher social-economic class [4].

\section{The Reason behind Dog}

The inspiration behind comparative oncology is simple. If a model is very similar to human beings then why can't we investigate it to introduce quality improvement for human healthcare? Dogs get many cancers that strike humans, including lymphoma, breast cancer and bone cancer. Contrasting to genetically altered laboratory animals that we used to test potential new cancer treatments, dogs develop this disease naturally. We can measure the same things in dog that we do in humans, like heart changes, organ function and blood pressure. Hence, these data can be applied directly to human diseases. With dogs, we can ask many questions that one cannot ask in mouse preclinical models of cancer and cannot answer in 
human clinical trials.

Treating naturally occurring diseases of dogs does not create an ethical issue as seen with experimentally induced disease in models and hence it provides a more robust model. The physiology of dog allows responding to drug metabolism in way comparable to humans, therefore the dog is used routinely for pharmaceutical toxicological studies [5]. Cancer is the most recurrent canine disease. Dogs develop spontaneous tumors that display behavior and histo-pathological characteristics to human tumors [6-8]. For example, in dogs, studying about the role of inhibition of telomere size shortening plays in cell immortalization and proliferation is relevant, because dog chromosomal telomeres closely resemble human telomeres than murine telomeres [9]. Among the practical benefits of clinical investigation of cancer in dogs is the fact that due to their shorter life span, clinical intervention is easily studied over a condensed period of time. The dog cancer cases have the survival rate over one year, rather than five, as in human oncology [8], thus the possibility to attain comparatively rapid results when performing clinical trials and monitoring disease progression in dogs.

\subsection{Why Not Mouse as Models?}

Murine models have been used in many studies, but in vivo murine model such as xenografts and transgenics fail to reiterating essential features and characteristics (such as heterogeneity, tumor microenvironment and dependence on steroid hormones) of human breast cancer $[10,11]$. In addition to the inherent evolutionary remoteness between mice and humans, further differences can originate due to induced genetic modifications (transgenic mice) or from altered presence of adjacent normal tissue, stromal cells, vasculature and immune system components (xenografts) [12-16]. Jointly these factors explain the limited values of murine model for studying cancer pathogenesis, progression and therapy and embody a major obstacle in identifying of reliable predictive molecular biomarkers and effective therapeutic agents development $[10,17]$.

\subsection{Studying Cancer with Dog Models}

Of all the disorders for which dogs are likely to enlighten human health, canine cancer is likely to have the greatest effect [18]. Cancer is the most frequent cause of diseaseassociated death in canines, and naturally occurring cancers have been well described in several breeds [19-21]. Although, substantial effort has been spent in studying common cancers, the dog has always serves as a model for rare tumors studies, including aggressive histiocytic sarcoma and lethal dendritic-cell neoplasms [22].

A mammary tumor is the common kind of tumor in unspayed female dogs. Similarly, breast cancer is the most widespread cancer in women, striking 1/8th women during their lifetime. Not all dogs or even women succumb to the disease. Many individuals have benign slow growing tumors, which are curable. In other cancerous cells grow profusely and metastasize. But like in studies done in rats or mice, caner researchers don't induce caner in dogs. Instead, the cancer is so commonly found in these models that the researchers compassionately treat them naturally. Another reason why canines render help in studying mammary tumor is the fact that each female has eight to ten mammary glands thereby making it probable to study several tumors-each cropping up separately from the other and therefore genetically unique-in one individual. However, studying separate breast tumors in humans is usually not possible because it is rare for a woman to develop more than one spontaneous tumor in the breast [23].

\subsection{Need for More Predictive Preclinical Models}

Due to cancer complexity and limited knowledge available in this arena, many animal models fall short of predictive. The information we try to decipher from these models comes to a limited scope every now and then. Also, it can be said that even humans are not prognostic models when it come to cancer studies. When considering the evaluation of novel therapy in a species distinct from humans, it should be essential to ensure that questions asked in preclinical models can be answered and certify the interpretation of answers within the totality of information available [24]. Murine cancer models have proved to be useful for analyzing the complicated pathways involved in cancer initiation, promotion and progression. However, they have proved incapable in defining features occurring in humans like long latency periods, genetic instability, and heterogeneity of tumor cells. Most important of all; the complex biology of cancer, recurrence and metastasis, which is an outcome in human patients, fails to be explained in the conventional mouse models and in cancer drug development. Thus, the needs for additional models that represent the human disease in an enhanced way are needed [24].

According to leading cancer researchers, dogs have proved to be good research subjects as they develop the disease spontaneously, and many of the modern breeds have developed over the past few hundred years using restricted gene pools. Due to this selective breeding, the breed genetics have been preserved and has made some breeds more susceptible to certain cancers. These aspects along with high grade of similarity between dog and human genomes have helped the researchers to compare their genomes and study the evolutionary genetic changes associated with cancer. These factors, coupled with high degree of similarity between the genomes of 
dogs and humans, which provide the researchers with an opportunity to compare the genomes and study the evolutionary genetic changes associated with cancer [25].

Another basis for using them as study models deals with how human drug trials are conducted. The benefit would be to study and test the treatment strategies in canines with aggressive tumors and monitor how they respond to the treatment. In this way, the comparative oncologists can test new treatment ideas against early-stage of cancers and delivering the drugs just as they would ultimately be in cancer patients [25]. This means more effective treatments can be developed more quickly, with less adverse health risks, for human trials and ultimately viable human treatments. Dogs also have shorter life spans than humans (most unfortunate) meaning scientists can more quickly determine whether a prevention strategy or therapy has a good chance of improving human survival rates [18]. Now moving on from the physiological similarities to genetic level, the chance of studying health and disease in the dog (Canis lupus familiaris) was significantly expanded with the release of the first public draft of the canine genome [26-28]. This prospect was advanced further with the development of high throughput technologies, such as expression and SNP microarrays that are commercially available for the dog nowadays $[26,29,30]$.

Last many years showed the sequencing of entire dog genome ( $99 \%$ complete, $\sim 2.5$ billion base pairs) and the confirmation of its close similarity with the human genome [31]. For many gene families and those genes linked to cancer, the relationship between dog and human gene sequences is found to be much closer than any other counterparts [32]. When molecular cytogenetic analysis of canine tumor cells was carried out from hematological malignancies, it was revealed that the ancestral chromosomal aberrations were preserved in comparable cancers of human and dog [33,34]. Altered expression of ERBB2 and TP53 genes in mammary carcinomas is similar to the two species hence, proving alike roles in carcinogenesis and prospective use as prognostic indicators [35-37]. Similar mutations in oncogenes resulted in different cancer in humans and dogs, a study suggested [38]. For example, a similar mutation in KIT, a tyrosine kinase growth factor receptor, is recognized in both human gastrointestinal stromal tumors (GIST) and dog mast-cell cancers [38]. Likewise, the intratumoral (cell-to-cell) heterogeneity in human breast tumors also takes place in cognate dog tumors [39]. Thus the natural consequences of this heterogeneity that causes the deadly features of human cancers like acquired resistance to therapy, recurrence and metastasis.

For nearly two decades now, we have seen a rise in canine genome utilization to understand the genetic foundation of disorders that are difficult to unravel in humans
[40-42]. Their large size makes the canine families amenable to conventional linkage mapping. This has been well exemplified in the canine gene search for hereditary multifocal renal cystadenocarcinoma and nodular dermatofibrosis (RCND) in German shepherds [43]. Also the canine genome provided an accessible set of validated expression profiles for human gene expression on both oligonucleotide and cDNA array platforms which, benefited researchers with several datasets that were publicly available through web-based interactive analytical tools [26,44-46]. Using similar approach, the availability of online database of canine normal tissue gene expression profiles will help in silica analysis of canine diseases. This will promote more rigorous comparative genomic analysis for humans, rat and dog tissues [47]. These comparative oncology studies enable identification of common gene regulatory regions as well as evolutionary conserved gene expression networks. Remarkable similarities have been found during the analysis of canine and human orthologous gene expression in their respective matched tissues [48]. This tissue expression data demonstration has supported in expanding and redefining canine gene ontologies consequently allowing further robust assessment of biological functions. Common inherited human diseases occur due to complex interactions between multiple genes and environmental factors. Dogs share a common environment with man, so the selection of an authentic model for a multifactorial human disease becomes an easy choice.

\section{Genomic Detailing}

The position of the dog within mammalian evolutionary tree also makes it an important channel for comparative analysis of the human genome [47]. High prevalence of specific diseases that affect certain breeds suggests that there are limited numbers of loci underlying for each disease. This makes the genetic analysis potentially more traceable in dogs than humans [49]. Within the exception of human, dog is the most intensely studied animal in medical practice, with detailed family history and pathology data [50]. Using genetic resources developed over the past 15years [51-56], researchers have already identified mutations in genes underlying 25 Mendelian diseases [57,58]. Dog is equally important for the comparative analysis of mammalian genome biology and evolution [47]. The important findings, after studying the dog's genome, its gene evolution, haplotype structure and phylogenetic etc showed the average transposon insertion rate of dog genome lower then humans. Also further comparison showed that $\sim 5.3 \%$ of the human genome contained functional elements that have been under purifying selection in both lineages. Similar patterns of evolution were observed in functionally related gene sets in human and dog lineages [47]. Unique unambiguous 
aligned sequences created multi-species synteny maps showed regions of conserved synteny between dog and human genome. The total number of breakpoints in humans was found to be substantially lower than that in dogs while more intra-chromosomal breakpoints were found in human lineages than dogs [47]. Although, the level of genomic rearrangement has been much higher in rodent than in human, comparison with dog shows that there are regions where the opposite is true. Human chromosome 17 is rich in segmental duplications and gene families, which may contribute to its genomic fragility [48].

\section{Gene Expression Profiles of Metastatic Canine Mammary Carcinomas Overlaps with Human Breast Cancer Profile}

Molecular mechanisms behind lymph node and distant metastasis in Canines still remain incomplete. Several studies have been done trying to cover this issue but significant metastasis associated and predictable expression patterns of single genes have still not been identified in canine mammary tumor (CMT) [35,59-61]. Global gene expression profiles that compare metastasizing versus non-metastasizing CMT are unavailable, whereas several studies on human breast cancer found to be significant metastasis associated expression profiles. The latter studies have identified several non-overlapping expression signatures, which are related to the development of lymph node and distant metastases and worse prognosis [6265]. Moreover, comparison of canine and human expression profiles disclosed an overlap of deregulated genes in human and canine mammary tumors [66].

The greatest challenge faced by clinical scientists is the incomplete understanding of genetic basis for complex human disease [62]. Regardless of numerous technological advances in genetics, the progress in this field has been slow due to the intricate gene-gene interactions and poorly understood environmental effects [67]. Also, the identification of these interactions and environmental influences is difficult to scrutinize in humans due to high level of genetic heterogeneity [68]. Many genome-wide association studies (GWAS) identified a small fraction of genetic basis of complex diseases [69]. And yet disease heritability is critical to understanding disease risk, the effects of environment and lifestyle on disease development, and response to treatment.

In one of the studies conducted by Klofleisch et al., a gene expression profile in CMT associated with early metastatic spread to lymph node was identified that could discriminate carcinomas with similar histological features but different metastatic potential. This expression profile contained several enriched functional gene classes and had significant overlaps with expression profiles of metastatic human breast cancer [35]. Med gene literature mining also confirmed the similarities found between canine and human mammary tumors in terms of increased proliferation, altered cell differentiation status and decreased cell adhesion [59].

Approximately $25 \%$ of the deregulated genes in metastatic canine carcinomas were cited in association with human breast cancer. In this subset of cited genes, a significant enrichment of genes associated with cell cycle regulation, protein kinases, DNA integrity checkpoint and protein metabolism was observed [61]. It was therefore likely that gene expression profiles may also predict metastasis in CMT. Klofleisch et al. concluded that metastatic spread of CMT to the lymph nodes was associated with a gene expression profile of increased cell cycle progression, altered cell differentiation and decreased growth factor signaling. Several key characteristic of metastasis associated gene expression are therefore similar between human and canine mammary tumors [35].

\section{Conclusions}

Comparative oncology has proved to be useful for clinico-pathological and therapeutic study. It has been proved beneficial to analyze the particular disease pattern between two or more than two species. The comparative oncology program has also focused on genetic history and the pattern of inheritance in different species and their correlation. This in turn has proved useful to the research workers to go into the depth of a particular study of interest.

The goal of scientific research has been to better understand cancer biology in order to understand cancer diagnosis, treatment and prevention in humans and animals. It is due to the exchange of ideas and observations between researchers studying human and companion animal cancer that these studies have become more common and successful.

Clinical and translational studies in dogs don't face the same constraints as human trials and hence a platform is prepared by previous researchers to further continue and enhance these studies for human betterment. We tried to sum up previous discoveries and conclusions that were done by renowned researchers. The focus is to analyze till where have we succeeded in enabling ourselves utilize this model for prognostic cancer strategies in humans. With comparative oncology studies researchers have become more confident in development and validation of new medical devices. Dog models showed that helical tomotherapy devices could successfully image, position, and treat spontaneously occurring tumors [70].

The evolutionary history of dogs, their position as a family member in many households, and the high level of health care they receive in our world offer tremendous opportunities. Alongside this combined with recently developed genetic resources, makes dogs outstanding 
models for the studying known genetic pathways, discovery of genetic and environmental contributions to disease, and translational studies in cancer risk, prevention, and treatments [70]. Increased gratitude of the inimitable and comparative values of dog as a model for diverse human diseases should promote and accelerate more researches leading to new better treatments and improved health care for both mankind and his best friend-the dog. Thus in conclusion our summation is that Dogs are exceptionally suited for use as an animal model of complex human disease due to their phenotypic diversity and naturally occurring disease resemblance to human conditions.

\section{REFERENCES}

[1] B. Leder, "Breast Cancer in Dogs,” 2009. http://www.thebark.com/content/breast-cancer-dogs

[2] J. Ferlay J, H. R. Shin, F. Bray, D. Forman, C. Mathers and D. M. Parkin, "Globcan 2008 v2.0, Cancer Incidence and Mortality Worldwide: IARC Cancer Base No. 10," 2010. http://globocan.iarc.fr, accessed on day/month/year

[3] Marisa Weiss, et al., "US Breast Cancer Statistics,” 2012. http://www.breastcancer.org/symptoms/understand_bc/sta tistics

[4] M. H Tirgan, “A Short Review on Breast Cancer,” 19962011. http://www.tirgan.com/brstcan.htm

[5] D. Smith, C. Broadhead, G. Descotes, et al., "Preclinical Safety Evaluation Using Nonrodent Species: An Industry/Welfare Project to Minimize Dog Use," The ILAR Journal, Vol. 43, Supplement S39-S42, 2002, pp. 39-42.

[6] E. G. McEwen, "Spontaneous Tumors in Dogs and Cats: Models for the Study OF Cancer Biology and Treatment," Cancer and Metastasis Reviews, Vol. 9, No. 2, 1990, pp. 125-136. doi:10.1007/BF00046339

[7] K. A. Hahn, L. Bravo, W. H. Adams and D. L. Frazier, "Naturally Occurring Tumors in Dogs as Comparative Models for Cancer Therapy Research,” In Vivo, Vol. 8, No. 1, 1994, pp. 133-143.

[8] D. M. Vail and E.G. McEwen, "Spontaneously Occurring Tumors of Companion Animals as Models for Human Cancer," Cancer Investigation, Vol. 18, No. 18, 2000, pp. 781-792. doi:10.3109/07357900009012210

[9] L. Nasir, P. Devlin, T. Mckevitt, et al., "Telomere Lengths and Telomerase Activity in Dog Tissues: A Potential Model System to Study Human Telomere and Telomerase Biology,” Neoplasia, Vol. 3, No. 4, 2001, pp. 351-359. doi:10.1038/sj.neo.7900173

[10] T. Vargo-Gogola and J. Rosen, "Modeling Breast Cancer: One Size Does Not Fit All,” Nature reviews Cancer, Vol. 7, No. 9, 2007, pp. 659-672. doi:10.1038/nrc2193

[11] S. Nandi, R. C. Guzman and J. Yang, "Hormones and Mammary Carcinogenesis in Mice, Rats, and Humans: A Unifying Hypothesis," Proceedings of the National Academy of Sciences of the United States of America, Vol. 92, No. 9, 1995, pp. 3650-3657. doi:10.1073/pnas.92.9.3650

[12] F. Balkwill, K. A. Charles and A. Mantovani, "Smolder- ing and Polarized Inflammation in the Initiation and Promotion of Malignant Disease," Cancer Cell, Vol. 7, No. 3, 2005, pp. 211-217. doi:10.1016/j.ccr.2005.02.013

[13] S. Goswami, E. Sahai, J. B. Wyckoff, M. Cammer, D. Cox, F. J. Pixley, E. R. Stanley, J. E. Segall and J. S. Condeelis, "Macrophages Promote the Invasion of Breast Carcinoma Cells via a Colony Stimulating Factor-1/Epidermal Growth Factor Paracrine Loop,” Cancer Research, Vol. 65, No. 12, 2005, pp. 5278-5283. doi:10.1158/0008-5472.CAN-04-1853

[14] K. L. Schwertfeger, J. M. Rosen and D. A. Cohen, "Mammary Gland Macrophages: Pleiotropic Functions in Mammary Development," Journal of Mammary Gland Biology and Neoplasia, Vol. 11, No. 3-4, 2006, pp. 229238.

[15] J. W. Pollard, "Role of Colony-Stimulating Factor-1 in Reproduction and Development," Molecular Reproduction and Development, Vol. 46, No. 1, 1997, pp. 54-60. doi:10.1002/(SICI)1098-2795(199701)46:1<54::AID-MR D9>3.0.CO;2-Q

[16] R. C. Hovey, T. B. McFadden and R. M. Akers, "Regulation of Mammary Gland Growth and Morphogenesis by the Mammary Fat Pad: A Species Comparison,” Journal of Mammary Gland Biology and Neoplasia, Vol. 4, No. 1, 1999, pp. 53-68. doi:10.1023/A:1018704603426

[17] N. Erin, W. Zhao, J. Bylander, G. Chase and G. Clawson, "Capsaicin-Induced Inactivation of Sensory Neurons Promotes a More Aggressive Gene Expression Phenotype in Breast Cancer Cells,” Breast Cancer Research and Treatment, Vol. 99, No. 3, 2006, pp. 351-364. doi:10.1038/nbt0906-1065b

[18] C. Khanna, K. Lindblad-Toh, D. Vail, C. London, P. Bergman, et al., "The Dog as a Cancer Model," Nature Biotechnology, Vol. 24, No. 9, 2006, pp. 1065-1066. doi:10.1038/nbt0906-1065b

[19] A. L. Shearin and E. A. Ostrander, "Leading the Way: Canine Models of Genomics and Disease,” Disease Models \& Mechanisms, Vol. 3, No. 1-2, 2010, pp. 27-34. doi:10.1242/dmm.004358

[20] R. T. Bronson, "Variation in Age at Death of Dogs of Different Sexes and Breeds," American Journal of Veterinary Research, Vol. 43, No. 11, 1982, pp. 2057-2059.

[21] L. E. Maquat, "Defects in RNA Splicing and the Consequence of Shortened Translational Reading Frames," The American Journal of Human Genetics, Vol. 59, No. 2, 1996, pp 279-286.

[22] C. Zandonella, "Cancer Collaboration Could Someday Help Dogs and Their Humans,” 2012.

https://www.princeton.edu/main/news/archive/S33/61/61 S27/

[23] I. K. Gordon and C. Khanna, "Modeling Oppurtunities in Comparative Oncology for Drug Development," The ILAR Journal, Vol. 51, No. 3, 2010, pp. 214-220. doi:10.1093/ilar.51.3.214

[24] J. Modiano, "Genetic Cancer Link between Humans and Dogs Discovered,” 2008. http://www.sciencedaily.com/releases/2008/02/08022811 2011.htm

[25] J. David, W. Waters and K. Wildasin, “The Vital Impor- 
tance of Comparative Oncology: Cancer Clues from Dogs,” 2006. http://landofpuregold.com/cancer/cop.htm

[26] K. Lindblad-Toh, C. M. Wade, T. S. Mikkelsen, E. K. Karlsson, D. B. Jaffe, et al., "Genome Sequence, Comparative Analysis and Haplotype Structure of the Domestic Dog,” Nature, Vol. 438, No. 7069, 2005, pp. 803-819. doi:10.1038/nature04338

[27] H. G. Parker and E. A. Ostrander, "Canine Genomics and Genetics: Running with the Pack,” PLOS Genetics, Vol. 1, No. 5, 2005, p. e58. doi:10.1371/journal.pgen.0010058

[28] J.A. Holzwarth, R. P. Middleton, M. Roberts, R. Mansourian, F. Raymond, et al., "The Development of a HighDensity Canine Microarray,” Journal of Heredity, Vol. 96, No. 7, 2005, pp. 817-820.

[29] M. Paoloni and C. Khanna, "Translation of New Cancer Treatments from Pet Dogs to Humans," Nature Reviews Cancer, Vol. 8, No. 2, 2008, pp. 147-156. doi:10.1038/nrc2273

[30] R. Thomas, A. Scott, C. F. Langford, S. P. Fosmire, C. M. Jubala, et al., "Construction of a 2-Mb Resolution BAC Microarray for CGH Analysis of Canine Tumors," Genome Research, Vol. 15, No. 12, 2005, pp. 1831-1837. doi:10.1101/gr.3825705

[31] M. M. Hoffman and E. Birney, "Estimating the Neutral Rate of Nucleotide Substitution Using Introns," Molecular Biology and Evolution, Vol. 24, No. 2, 2007, pp 522531. doi:10.1101/gr.3825705

[32] M. Breen and J. F. Modiano, "Evolutionarily Conserved Cytogenetic Changes in Hematological Malignancies of Dogs and Humans-Man and His Best Friend Share More Than Companionship," Chromosome Research, Vol. 16, No. 1, 2008, pp. 145-154. doi:10.1007/s10577-007-1212-4

[33] R. Thomas, K. C. Smith, E. A. Ostrander, F. Galibert and M Breen, "Chromosome Aberrations in Canine Multicentric Lymphomas Detected with Comparative Genomic Hybridization and a Panel of Single Locus Probes,” British Journal of Cancer, Vol. 89, No. 8, 2003, pp. 15301537. doi:10.1038/sj.bjc.6601275

[34] A. Rungsipipat, S. Tateyama, R. Yamaguchi, K. Uchida, N. Miyoshi and T. Hayashi, "Immunohistochemical Analysis of c-yes and c-erbB-2 Oncogene Products and p53 Tumor Suppressor Protein in Canine Mammary Tumors," Journal of Veterinary Medical Science, Vol. 61, No. 1, 1999, pp. 27-32. doi:10.1292/jvms.61.27

[35] A. Setoguchi, T. Sakai, M. Okuda, K. Minehata, M. Yazawa, T. Ishizaka, T. Watari, R. Nishimura, N. Sasaki, A. Hasegawa and H. Tsujimoto, "Aberrations of the p53 Tumor Suppressor Gene in Various Tumors in Dogs," American Journal of Veterinary Research, Vol. 62, No. 3, 2001, pp. 433-439. doi:10.2460/ajvr.2001.62.433

[36] S. Haga, M. Nakayama, K. Tatsumi, M. Maeda, S. Imai, S. Umesako, H. Yamamoto, J. Hilgers and N. H. Sarkar, "Overexpression of the p53 Gene Product in Canine Mammary Tumors,” Oncology Reports, Vol. 8, No. 6, 2001, pp. 1215-1219.

[37] K. Ozaki, T. Yamagami, K. Nomura and I. Narama, "Mast Cell Tumors of the Gastrointestinal Tract in 39 Dogs,” Veterinary Pathology, Vol. 39, No. 5, 2002, pp.
557-564. doi:10.1354/vp.39-5-557

[38] A. Porrello, P. Cardelli and E. P. Spugnini, “Oncology of Companion Animals as a Model for Humans-An Overview of Tumor Histotypes," Journal of Experimental \& Clinical Cancer Research, Vol. 25, No. 1, 2006, pp. 97105.

[39] K. L. Tsai, L. A. Clark and K. E. Murphy, "Understanding Hereditary Diseases Using the Dog and Human as Companion Model Systems,” Mammalian Genome, Vol. 18, No. 6-7, 2007, pp. 444-451. doi:10.1007/s00335-007-9037-1

[40] E. K. Karlsson and K. Lindblad-Toh, "Leader of the Pack: Gene Mapping in Dogs and Other Model Organisms," Nature Reviews Genetics, Vol. 9, No. 9, 2008, pp. 713725. doi:10.1038/nrg2382.

[41] E. A. Ostrander and E. Giniger, “Semper Fidelis: What Man's Best Friend Can Teach Us about Human Biology and Disease," The American Journal of Human Genetics, Vol. 61, No. 3, 1997, pp. 475-480. doi:10.1086/515522

[42] T. J. Jónasdóttir, C. S. Mellersh, L. Moe, et al., "Genetic Mapping of a Naturally Occurring Hereditary Renal Cancer Syndrome in Dogs," Proceedings of the National Academy Sciences of the United States of America, Vol. 97, No. 8, 2000, pp. 4132-4137. doi:10.1073/pnas.070053397

[43] L. L. Hsiao, F. Dangond, T. Yoshida, R. Hong, R. V. Jensen, et al., "A Compendium of Gene Expression in Normal Human Tissues,” Physiol Genomics, Vol. 7, No. 2, 2001, pp. 97-104.

[44] A. Saito-Hisaminato, T. Katagiri, S. Kakiuchi, T. Nakamura, T. Tsunoda, et al., "Genome-Wide Profiling of Gene Expression in 29 Normal Human Tissues with a cDNA Microarray,” DNA Research, Vol. 9, No. 2, 2002, pp. 35-45. doi:10.1093/dnares/9.2.35

[45] R. Shyamsundar, Y. H. Kim, J. P. Higgins, K. Montgomery, M. Jorden, et al., "A DNA Microarray Survey of Gene Expression in Normal Human Tissues,” Genome Biology, Vol. 6, No. 3, 2005, p. R22. doi:10.1186/gb-2005-6-3-r22

[46] G. Son, S. Bilke, S. Davis, B. T. Greer, J. S. Wei, et al., "Database of mRNA Gene Expression Profiles of Multiple Human Organs,” Genome Research, Vol. 15, No. 3, 2005, pp. 443-450. doi:10.1101/gr.3124505

[47] J. Briggs, M. Paoloni, Q. R. Chen, X. Wen, J. Khan and C. Khanna, "A Compendium of Canine Normal Tissue Gene Expression,” PLoS One, Vol. 6, No. 5, 2011, pp. 101-107. doi:10.1371/journal.pone.0017107

[48] E. A. Ostrander, F. Galibert and D. F. Patterson, "Canine Genetics Comes of Age,” Trends in Genetics, Vol. 16, No. 3, 2000, pp. 117-123. doi:10.1016/S0168-9525(99)01958-7

[49] D. Patterson, "Companion Animal Medicine in the Age of Medical Genetics,” Journal of Veterinary Internal Medicine, Vol. 14, No. 1, 2000, pp. 1-9. doi:10.1111/j.1939-1676.2000.tb01492.x

[50] M. Breen, et al., "Chromosome-Specific Single-Locus FISH Probes Allow Anchorage of an 1800 Marker Integrated Radiation-Hybrid/Linkage Map of the Domestic Dog Genome to All Chromosomes," Genome Research, 
Vol. 11, No. 10, 2001, pp. 1784-1795. doi:10.1101/gr.189401

[51] M. Breen, J. Bullerdiek and C. F. Langford, "The DAPI Banded Karyotype of the Domestic Dog (Canis Familiaris) Generated Using Chromosome-Specific Paint Probes,” Chromosome Research, Vol. 7, No. 5, 1999, pp. 401-406. doi:10.1023/A:1009224232134

[52] M. Breen, et al., “An Integrated 4249 Marker FISH/RH Map of the Canine Genome,” BMC Genomics, Vol. 5, 2004, p. 65. doi:10.1186/1471-2164-5-65

[53] C. Hitte, et al., "Facilitating Genome Navigation: Survey Sequencing and Dense Radiation-Hybrid Gene Mapping," Nature Reviews Genetics, Vol. 6, No. 8, 2005, pp. 643648. doi:10.1038/nrg1658

[54] R. Li, et al., "Construction and Characterization of an Eightfold Redundant Dog Genomic Bacterial Artificial Chromosome Library," Genomics, Vol. 58, No. 1, 1999, pp. 9-17. doi:10.1006/geno.1999.5772

[55] E. F. Kirkness, et al., “The Dog Genome: Survey Sequencing and Comparative Analysis," Science, Vol. 301, No. 5641, 2003, pp. 1898-1903. doi:10.1126/science.1086432

[56] N. Sutter and E. Ostrander, "Dog Star Rising: The Canine Genetic System,” Nature Reviews Genetics, Vol. 5, No. 12, 2004, pp. 900-910. doi:10.1038/nrg1492

[57] F. Galibert, C. Andre and C. Hitte, "Dog as a Mammalian Genetic Model [in French],” Medicine Science (Paris), Vol. 20, No. 8-9, 2004, pp.761-766.

[58] R. Klopfleisch, D. Lenze, M. Hummel and A. D. Gruber, "Metastatic Canine Mammary Carcinomas can be Identified by a Gene Expression Profile That Partly Overlaps with Human Breast Cancer Profiles,” BMC Caner, Vol. 10, 2010, p. 618. doi:10.1186/1471-2407-10-618

[59] A. Nieto, M. D. Perez-Alenza, N. Del Castillo, E. Tabanera, M. Castano and L. Pena, "BRCA1 Expression in Canine Mammary Dysplasias and Tumours: Relationship with Prognostic Variables," Journal of Comparative Pathology, Vol. 128, No. 4, 2003, pp. 260-268. doi:10.1053/jcpa.2002.0631

[60] S. K. Muthuswamy, D. Li, S. Lelievre, M. J. Bissell and J. S. Brugge, "ErbB2, But Not ErbB1, Reinitiates Proliferation and Induces Luminal Repopulation in Epithelial Acini,” Nature Cell Biology, Vol. 3, No. 9, 2001, pp. 785792. doi:10.1038/ncb0901-785

[61] E. S. Lander and N. J. Schork, "Genetic Dissection of Complex Traits,” Science, Vol. 265, No. 5181, 1994, pp.
2037-2048. doi:10.1126/science.8091226

[62] X. Lu, Z. C. Wang, J. D. Iglehart, X. Zhang and A. L. Richardson, "Predicting Features of Breast Cancer with Gene Expression Patterns,” Breast Cancer Research and Treatment, Vol. 108, No. 2, 2008, pp. 191-201. doi:10.1007/s10549-007-9596-6

[63] B. Weigelt, Z. Hu, X. He, C. Livasy, L. A. Carey, M. G. Ewend, A. M. Glas, C. M. Perou and L. J. Van’t Veer, "Molecular Portraits and 70-Gene Prognosis Signature Are Preserved throughout the Metastatic Process of Breast Cancer,” Cancer Research, Vol. 65, No. 20, 2005, pp. 9155-9158. doi:10.1158/0008-5472.CAN-05-2553

[64] L. J. Van't Veer, H. Dai, M. J. Van de Vijver, Y. D. He, A. A. Hart, M. Mao, H. L. Peterse, K. Van der Kooy, M. J. Marton, A. T. Witteveen, et al., "Gene Expression Profiling Predicts Clinical Outcome of Breast Cancer," Nature, Vol. 415, No. 6871, 2002, pp. 530-536. doi:10.1038/415530a

[65] P. Uva, L. Aurisicchio, J. Watters, A. Loboda, A. Kulkarni, J. Castle, F. Palombo, V. Viti, G. Mesiti, V. Zappulli, et al., "Comparative Expression Pathway Analysis of Human and Canine Mammary Tumors," BMC Genomics, Vol. 10, No. 135, 2009, p. 135. doi:10.1186/1471-2164-10-135

[66] K. Strauch, et al., "How to Model a Complex Trait. General Considerations and Suggestions," Human Heredity, Vol. 55, No. 4, 2003, pp. 202-210. doi:10.1159/000073204

[67] E. K. Karlsson and K. Lindblad-Toh, "Leader of the Pack: Gene Mapping in Dogs and Other Model Organisms," Nature Reviews Genetics, Vol. 9, No. 9, 2008, pp. 713725. doi:10.1038/nrg2382

[68] T. A. Manolio, et al., "Finding the Missing Heritability of Complex Diseases,” Nature, Vol. 461, No. 7265, 2009, pp. 747-753. doi:10.1038/nature08494

[69] M. Paoloni and C. Khanna, "Translation of New Cancer Treatments from Pet Dogs to Humans," Nature Reviews Genetics, Vol. 8, No. 2, 2008, pp. 147-156. doi:10.1038/nrc2273

[70] H. G. Parker, A. L. Shearin and E. A. Ostrander, "Man’s Best Friend Becomes Biology's Best in Show: Genome Analyses in the Domestic Dog," Annual Reviews of Genetics, Vol. 44, 2010, pp. 309-336. doi:10.1146/annurev-genet-102808-115200 